\title{
Incidencia de microcefalia neonatal en el Hospital Cayetano Heredia de Lima, 2016 - 2017.
}

Incidence of Neonatal microcephaly in the Hospital Cayetano Heredia, Lima, 2016 - 2017.

Cynthia Samaniego Lozano ${ }^{1,2, a}$, Carol Munayco Cortez ${ }^{1,2, b}$, Daniel Guillén-Pinto ${ }^{1,2, a}$

\section{RESUMEN}

Objetivo: Determinar la incidencia de microcefalia neonatal en el Hospital Cayetano Heredia durante un periodo definido. Material y Métodos: Se consideró como microcefalia un perímetro cefálico menor que el tercer percentil para la edad y el sexo. Se usaron los registros del Sistema Informático Perinatal del Hospital durante el periodo $1^{\circ}$ de enero, 2016 a 31 de diciembre, 2017. Los límites de normalidad fueron establecidos mediante el uso de parámetros de la Tabla de Fenton. Resultados: De un total de 416 casos de microcefalia neonatal, se registró una tasa general de 53,4 por mil nacidos vivos; en prematuros ( $<37$ semanas), la tasa fue de 185 por mil nacidos vivos, con niveles mayores en varones. Conclusiones: Se encontró una alta tasa de incidencia de microcefalia, mayor aún en prematuros, tasa probablemente vinculada a una causalidad múltiple, sin poder descartarse los sesgos observacionales.

PALABRAS CLAVE: Microcefalia, neonato, incidencia.

\section{SUMMARY}

Objective: To determine the incidence of neonatal microcephaly in the Hospital Cayetano Heredia, throughout a well-defined period. Material and Methods: Microcephaly was determined by a cephalic perimeter lower than the third percentile for age and sex. Reported records of the Hospital's Perinatal Computer System between January $1^{\text {st }}, 2016$ and December 31 ${ }^{\text {st }}$, 2017, were used. Fenton growth chart's parameters were used to determine the limits of normality. Results: A total of 416 cases of neonatal microcephaly were registered, a general rate of 53.4 per 1,000 live births, up to 185 per 1000 live births in preterm infants ( $<37$ weeks), with higher figures among males. Conclusions: A high incidence rate of microcephaly was found, probably higher in preterm infants and due to multiple causes. Observational biases cannot be discarded.

KEYWORDS: Microcephaly, neonate, incidence, preterm.

\section{INTRODUCCIÓN}

La microcefalia se define como el menor tamaño de la cabeza, identificada cuando el perímetro cefálico (PC) se encuentra menor al 3er percentil o menor de 2 DE para la edad y el sexo. El PC constituye uno de los mejores indicadores de neurodesarrollo en la etapa perinatal, porque se correlaciona con la maduración del tejido cerebral (1). Asimismo, su utilidad destaca para la vigilancia del desarrollo durante la primera infancia (2).

La prevalencia de microcefalia varía de acuerdo a los países, Europa reportó una tasa de 1.5 por 10 000 (3), la ECLAMC (Latin American Collaborative Study of Congenital Malformations) fue de 3,0 por

Facultad de Medicina, Universidad Peruana Cayetano Heredia. Lima, Perú.

Hospital Cayetano Heredia. Lima, Perú.

Neurólogo Pediatra

Médico Residente 
10000 (4) y la Organización Mundial de la Salud (OMS) estimó una proporción de 0,5 a $1 \%$ (5). La vigilancia de su prevalencia permite medir el efecto lesivo de potenciales agentes infecciosos, nutricionales y ambientales sobre el sistema nervioso fetal. Al respecto, en Latinoamérica, en el 2015, el Ministerio de Salud de Brasil, informó sobre un importante incremento de casos de microcefalia, inicialmente en el estado de Pernambuco y luego en otros estados de la región noreste de Brasil, relacionados con la infección por el virus Zika, cuyo impacto aún continúa en observación (6).

Nuestro país, siguiendo las recomendaciones de la Organización Panamericana de la Salud (OPS) y de la Organización Mundial de la Salud (7), en el 2016, implementó un Sistema de Vigilancia Centinela de Microcefalia (8), para responder a la demanda de los servicios especializados que atienden los síndromes congénitos, con especial interés en Zika. La implementación de estas medidas se encuentra en proceso.

La vigilancia de la prevalencia de microcefalia constituye un aspecto muy importante en sectores de la población con menores recursos económicos, expuestos a riesgos infecciosos o del medio ambiente. El objetivo de este trabajo fue analizar el registro de la microcefalia neonatal en el Hospital Cayetano Heredia durante los años 2016 y 2017.

\section{MATERIAL Y MÉTODOS}

Se realizó un estudio observacional descriptivo exploratorio, utilizando el Reporte del Hospital Cayetano Heredia de Lima (HCH) al Sistema
Informático Perinatal (SIP), del 1 de enero del 2016 hasta el 31 de diciembre del 2017.

Fueron incluidos los datos de todos los recién nacidos vivos que tuvieran registro del perímetro cefálico, de 24 a 40 semanas de edad gestacional. Se seleccionaron los neonatos que tuvieron perímetro cefálico inferior a 3er percentil $(<2 \mathrm{DS})$ para la edad gestacional y sexo.

Para determinar los límites inferiores del perímetro cefálico para neonatos prematuros y a término de $37 \mathrm{a}$ 40 semanas inclusive, se usó la Curva de Crecimiento de Fenton del año 2003, que permite determinar el crecimiento craneal desde las 22 semanas (9). Además, se registro el número de casos por sexo y edad gestacional.

El estudio contó con la aprobación del Comité de Ética del $\mathrm{HCH}$. Los datos fueron almacenados y depurados en una hoja de cálculo del programa Excel MicrosoftC), luego fueron procesados con el programa STATA (C) versión 13. Se aplicaron tablas de frecuencias absolutas y porcentuales, y se determinaron las tasas de incidencia. Se aplicó el riesgo relativo (RR) para confirmar si las variables de exposición conducían a un mayor riesgo de macrocefalia. En las pruebas de inferencia se utilizó un intervalo de confianza al 95\%.

\section{RESULTADOS}

Durante el periodo de estudio nacieron 8160 neonatos vivos, descontando las omisiones (80 neonatos) y fallecidos (101 neonatos), se hallaron 436 casos de microcefalia, 201 casos en el año 2016 y 235 en el año 2017, determinando una incidencia de 53,4 casos por cada mil nacidos vivos.

Tabla 1. Incidencia de microcefalia por años. HCH, 2016-2017

\begin{tabular}{cccccccccc}
\hline \multicolumn{1}{c}{$2016-2017$} & \multicolumn{9}{c}{2016} \\
\hline $\begin{array}{c}\text { EG } \\
\text { (sem) }\end{array}$ & $\mathbf{n}$ & Casos & Incidencia & $\mathbf{n}$ & Casos & Incidencia & $\mathbf{n}$ & Casos & Incidencia \\
$24-27$ & 41 & 36 & $87,8 \%$ & 21 & 16 & $76,2 \%$ & 20 & 20 & $100,0 \%$ \\
$28-29$ & 25 & 19 & $76,0 \%$ & 12 & 12 & $100,0 \%$ & 13 & 7 & $53,8 \%$ \\
$30-31$ & 57 & 23 & $40,4 \%$ & 30 & 13 & $43,3 \%$ & 27 & 10 & $37,0 \%$ \\
$32-33$ & 85 & 12 & $14,1 \%$ & 33 & 4 & $12,1 \%$ & 52 & 8 & $15,4 \%$ \\
$34-36$ & 614 & 62 & $10,1 \%$ & 275 & 28 & $10,2 \%$ & 339 & 34 & $10,0 \%$ \\
$37-38$ & 2,720 & 114 & $4,2 \%$ & 1,361 & 57 & $4,2 \%$ & 1,359 & 57 & $4,2 \%$ \\
$39-40$ & 4,618 & 170 & $3,7 \%$ & 2,238 & 71 & $3,2 \%$ & 2,380 & 99 & $4,2 \%$ \\
Total & 8,160 & 436 & $5,3 \%$ & 3,970 & 201 & $5,1 \%$ & 4,190 & 235 & $5,6 \%$ \\
\hline
\end{tabular}


En el 2017 la incidencia de microcefalia fue de $5,6 \%$ aumentando un $0,5 \%$ con respecto al 2016. Se observa una relación inversa entre la incidencia de microcefalia y la edad gestacional (tabla 1).

La tasa de incidencia de microcefalia en los prematuros fue de 185 por mil nacidos vivos y entre los niños a término la incidencia fue 39 casos por cada mil nacidos vivos.

En el gráfico 1 se muestra la incidencia de microcefalia según sexo y edad gestacional, y se observa que, antes de las 32 semanas de edad gestacional, en los varones es más frecuente, posteriormente, desaparece esta diferencia.
En el grupo de bebés mujeres con microcefalia, el $33,2 \%$ fueron prematuras, en cambio en el grupo de bebés mujeres sin microcefalia el porcentaje de prematuridad disminuyó a 8,8\%. En el grupo de varones con microcefalia, el 36,3\% fueron prematuros, y este porcentaje disminuyó a $8,5 \%$ en el grupo de varones sin microcefalia. Tanto en mujeres como en varones las diferencias del porcentaje de prematuridad fueron muy significativas $(\mathrm{p}<0,001)$.

El riesgo relativo de microcefalia, en el caso de los varones prematuros fue mayor que el de las mujeres, 5,1 veces, es decir, que un varón prematuro tendrá 5 veces más riesgo de microcefalia con respecto a otro varón que nació a término; por lo tanto, los varones prematuros tienen más riesgo de microcefalia que las mujeres prematuras (tabla 2).

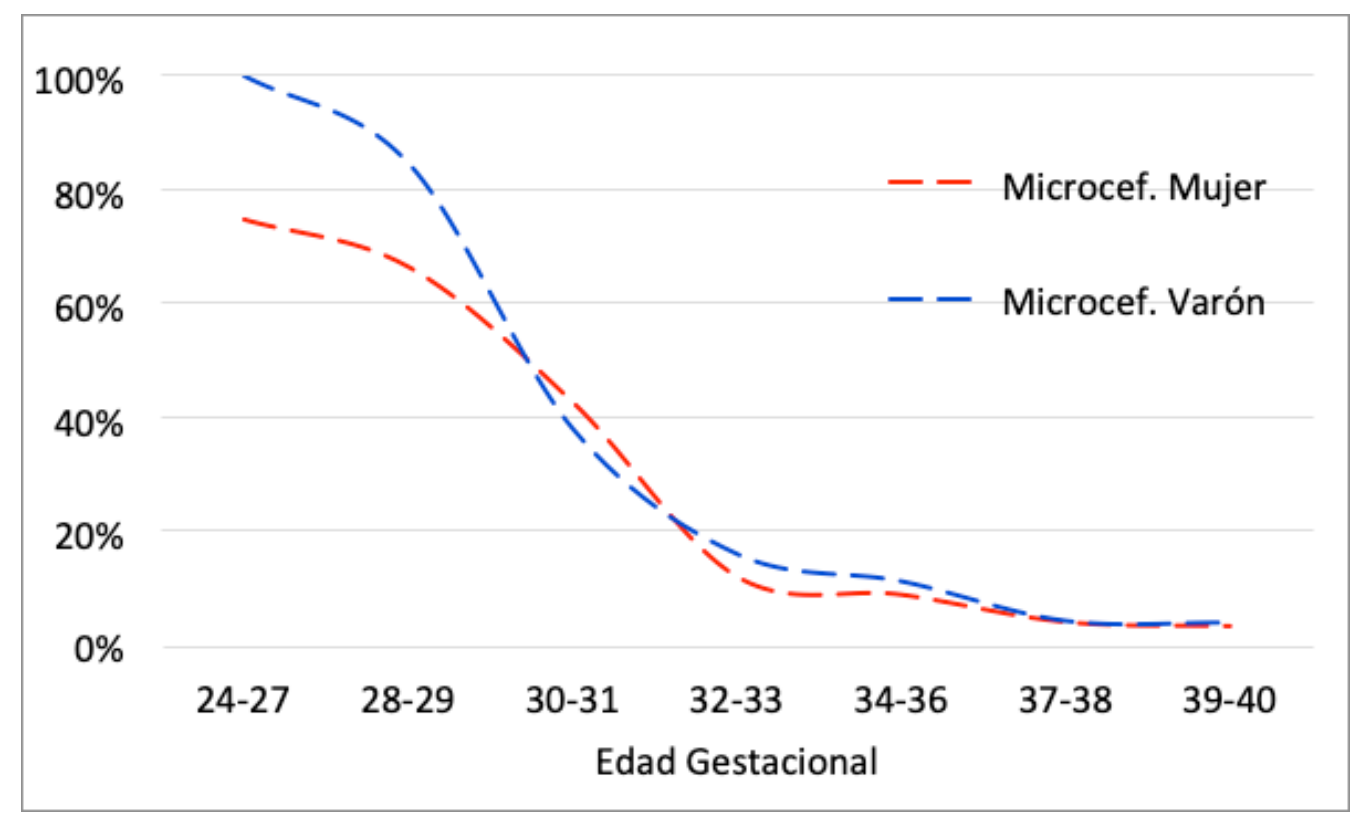

Gráfico 1. Incidencia de microcefalia por edad gestacional y sexo

(HCH, 2016-2017)

Tabla 2. Riesgo de microcefalia de acuerdo a la edad gestacional y sexo. HCH, 2016-2017

\begin{tabular}{llrrrrrr}
\hline \multirow{2}{*}{ Sexo } & Edad & \multicolumn{2}{c}{$\mathrm{PC}<\mathrm{P3} *$} & $\mathrm{PC} \geq \mathrm{P} 3$ & $\mathrm{RR}$ & $\mathrm{I}$ IC95\% \\
& gestacional & $\mathrm{n}$ & $\%$ & $\mathrm{n}$ & $\%$ & & \\
\hline \multirow{2}{*}{ Mujer } & Prematuras & 67 & 33,2 & 333 & 8,8 & 4,4 & $3,4-5,8$ \\
& A término & 135 & 66,8 & 3,445 & 91,2 & 1 & \\
& Prematuros & 85 & 36,3 & 337 & 8,5 & 5,1 & $4,0-6,5$ \\
\multirow{2}{*}{ Varón } & A término & 149 & 63,7 & 3,609 & 91,5 & 1 & \\
& Arematuros & 152 & 34,8 & 670 & 8,7 & 4,8 & $4,0-5,7$ \\
& Ambos & 284 & 65,1 & 7054 & 91,3 & 1 & \\
& A término & 28 & & & &
\end{tabular}

*PC=perímetro cefálico, $\mathrm{P} 3=$ percentil 3 


\section{DISCUSIÓN}

La vigilancia de microcefalia cobró importancia, a raíz de un informe epidemiológico brasilero de noviembre del 2015, el cual informó sobre 399 casos de microcefalia en recién nacidos de siete estados del Noreste de Brasil, derivando en una asociación con la infección por el virus Zika (10).

Posteriormente, se presentaron varios estudios que tenían como objetivo determinar esta relación con la prevalencia de microcefalia. Entre ellos, Nunes et al. que sugieren una relación temporal entre el aumento de la notificación de casos de microcefalia y el brote de virus Zika, principalmente en el Noreste de Brasil (2). Otro informe, de Magalhães-Barbosa et al, describen la prevalencia de microcefalia en los bebés ingresados en las unidades de cuidados intensivos neonatales (UCIN) en ocho hospitales privados en el sureste y el medio oeste de Brasil, de 2011 a 2015, de 8275 neonatos la prevalencia general de microcefalia fue del 5,6\%, mayor en los neonatos extremadamente prematuros (11).

El Perú siguiendo las recomendaciones de la Organización Panamericana de la Salud, viene implementando el Sistema de Vigilancia de Microcefalia en 30 hospitales, para lo cual el despacho Viceministerial de Salud Pública aprobó el Protocolo Sanitario de Urgencia RVM 014-2016SA, cuyo objetivo es establecer las pautas para la vigilancia epidemiológica de microcefalia. En la pesquisa bibliográfica nacional se encontró un estudio retrospectivo que analizó 33 casos de microcefalia en el servicio de neurocirugía del Hospital del Niño (12); no se ha podido encontrar otras referencias locales. Por tal motivo, despierta nuestro interés analizar el registro de la microcefalia neonatal en el Hospital Cayetano Heredia, que mantiene un óptimo reporte neonatal.

La incidencia de 5,3\% de microcefalia, está muy por arriba de las cifras de prevalencia que estima la OMS de 0,5 a $1 \%$, esta variación estaría en concordancia con otros estudios que demostraron un incremento en el número de casos de microcefalia desde finales del año 2015, en el contexto de infección por el virus Zika, sobretodo en Brasil (13). Por otro lado, existen factores que también podrían explicar esta diferencia, tales como el orden demográfico poblacional, técnica de medición, elección de los instrumentos de medición, aplicación de criterios para definir la microcefalia y mayor prevalencia de infecciones intrauterina entre las que destaca la infección por citomegalovirus, puntos que han sido objeto de un amplio debate, especialmente en relación con los recién nacidos prematuros (14). En este estudio, usamos la curva de Fenton, la cual armoniza con el estándar de crecimiento de la OMS en niños prematuros $(9,15)$.

En comparación con los recién nacidos a término, los prematuros presentaron una mayor incidencia de microcefalia, ligeramente mayor en el sexo masculino. Esta diferencia podría ser explicada porque los prematuros son más afectados por los factores que condicionan microcefalia, entre ellos los genéticos: trastornos cromosómicos-metabólicos, y las etiologías no genéticas: congénitas, exposición intrauterina a teratógenos, lesiones perinatales y desnutrición severa (16).

Las limitaciones del estudio fueron las que corresponden a un estudio de observación retrospectiva. Sin embargo, las fortalezas son haber mostrado una tasa de incidencia hospitalaria que servirá como marco referencial para hospitales de tercer nivel, para niños prematuros y a término.

En conclusión, se encontró una incidencia de 53,4 casos por cada mil nacidos vivos. Mayor en prematuros, y con predominio de los varones. Esperamos que este análisis fomente el desarrollo de protocolos de diagnóstico, basados en una medida correcta del perímetro cefálico al nacer, el examen clínico, estudios de laboratorio y de neuroimagen como la ecografía cerebral. En vista de que la microcefalia se asocia con un alto riesgo de morbilidad y afección del neurodesarrollo.

Fuentes de financiamiento: Autofinanciado.

Conflictos de interés: Los autores no reportan ningún conflicto de interés.

\section{Correspondencia:}

Cynthia Luz Samaniego Lozano

Correo electrónico: cynthia.samaniego85@gmail.com

\section{REFERENCIAS BIBLIOGRÁFICAS}

1. Dolk $\mathrm{H}$. The predictive value of microcephaly during the first year of life for mental retardation at seven years. Dev Med Child Neurol. 1991; 33(11): 974983. DOI: $10.1111 / j .1469-8749.1991 . t b 14813 . x$

2. Figueiras A. Manual para la vigilancia del desarrollo infantil en el contexto de AIEPI. $2^{\mathrm{a}}$ ed. Washington 
DC: OPS; 2011. p. 3-45.

3. Morris JK, Rankin J, Garne E, Loane M, Greenlees $\mathrm{R}$, Addor M-C, et al. Prevalence of microcephaly in Europe: population based study. BMJ. 2016; 354:i4721. doi: 10.1136/bmj.i4721.

4. Orioli IM, Dolk H, Lopez-Camelo JS, Mattos D, Poletta FA, Dutra MG, et al. Prevalence and clinical profile of microcephaly in South America pre-Zika, 2005-14: prevalence and case-control study. BMJ. 2017;359:j5018. doi: 10.1136/bmj.j5018.

5. Nunes ML, Carlini CR, Marinowic D. Microcephaly and Zika virus: a clinical and epidemiological analysis of the current outbreak in Brazil. J Pediatr (Rio J). 2016; 92(3): 230-240.

6. European Centre for Disease Prevention and Control. Rapid risk assessment - Zika virus epidemic in the Americas: potential association with microcephaly and Guillain-Barré syndrome. Solna, Sweden: European Centre for Disease Prevention and Control First update; 2016. (Citado el 21 de enero del 2019) Disponible en: https://ecdc.europa.eu/en/ publications-data/rapid-risk-assessment-zika-virusdisease-epidemic-potential-association-4

7. Organización Panamericana de la Salud. Lineamientos preliminares de vigilancia de microcefalia en recién nacidos en entornos con riesgo de circulación de virus Zika. Washington DC: Organización Panamericana de la Salud; 2016.

8. Ministerio de Salud. Protocolo Sanitario de Urgencia: Vigilancia Centinela de Microcefalia. Informe $\mathrm{N}^{\circ}$ 221-2016-OGAJ/MINSA. Lima: Ministerio de Salud; 2016.

9. Fenton T, Kim J. A systematic review and metaanalysis to revise the Fenton growth chart for preterm infants. BMC Pediatrics. 2013; 13:59.

10. Ministerio de Salud. Informe del Centro de Operaciones de Emergencias en Salud Publica sobre microcefalias. Semana Epidemiológica 47 de 2015. Brasilia: Ministerio de Salud de Brasil; 2015.
11. De Magalhães MC, Prata A, Robaina JR. Prevalence of microcephaly in eight south-eastern and midwestern Brazilian neonatal intensive care units: 2011-2015. Arch Dis Child. 2017; 102(8): 728-734.

12. Effio H, Cubas R, Pozadas G, Pizarro M. Microcefalia: estudio retrospectivo en el Hospital del Niño-Lima / Microcephaly: retrospective study in Hospital del Niño-Lima. Diagnóstico Perú. 1987;20(1):18-24.

13. Quintó L, García-Basteiro AL, Bardají A, González R, Padilla N, Martinez-Espinosa FE, et al. The challenge of assessing microcephaly in the context of the Zika Virus Epidemic. Journal of Tropical Pediatrics. 2017;63(6): 495-498. DOI: https://doi. org/10.1093/tropej/fmx015

14. Ashwal S, Michelson D, Plawner L, Dobyns WB. Practice Parameter: Evaluation of the child with microcephaly (an evidence-based review) Report of the Quality Standards Subcommittee of the American Academy of Neurology and the Practice Committee of the Child Neurology Society. Neurology. 2009; 73(11): 887-897.

15. Villar J, Giuliani F, Bhutta ZA. Postnatal growth standards for preterm infants: the Preterm Postnatal Follow-up Study of the INTERGROWTH-21st Project. Lancet Glob Health. 2015; 3(11): 681-691. doi: 10.1016/S2214-109X(15)00163-1

16. World Health Organization. Screening, assessment and management of neonates and infants with complications associated with Zika virus exposure in utero. Geneva: World Health Organization; 2016. (Fecha de acceso 10 de setiembre del 2019) Disponible en: http://apps.who.int/iris/bitstream/10665/204475/ 1/WHO_ZIKV_MOC_16.3_eng. pdf

Recibido: 06/06/2019

Aceptado: 13/09/2019 